\title{
Prevención de la transmisión vertical de VIH en mujeres migrantes internacionales: Escenario actual y desafíos
}

\author{
Prevention of vertical transmission of HIV in international migrant women: \\ Current scenario and challenges
}

\author{
Báltica Cabieses ${ }^{\mathrm{a}, \mathrm{b}, \mathrm{c}}$, Camila Sepúlvedaa,d, Alexandra Obach ${ }^{\mathrm{a}, \mathrm{e}}$ \\ aPrograma de Estudios Sociales en Salud, Facultad de Medicina Clínica Alemana, Universidad del Desarrollo. Santiago, Chile. \\ bDepartament of Health Sciences. University of York, York, United Kingdom. \\ 'Enfermera-Matrona. \\ dMatrona. \\ eAntropóloga.
}

Recibido: 10 de febrero de 2020; Aceptado: 15 de julio de 2020

¿Qué se sabe del tema que trata este estudio?

La prevención de la transmisión vertical de VIH en migrantes gestantes es una prioridad de interés nacional e internacional. Esfuerzos de diversos actores sociales y de salud han intentado aportar a esta problemática, en favor del cumplimiento de metas sanitarias.

\begin{abstract}
¿Qué aporta este estudio a lo ya conocido?
El documento aporta con una revisión integradora del desafío de la prevención de transmisión vertical de VIH en migrantes gestantes, discutiendo acciones desarrolladas y promoviendo adaptaciones interculturales que reconozcan la diversidad social y cultural de esta población en Chile.
\end{abstract}

\section{Resumen}

La prevención de la transmisión vertical de VIH es un desafío para todos los países del mundo. Esto se ve complejizado por la construcción permanente de sociedades globales, con grado variable de población migrante internacional. Las políticas, programas y acciones sanitarias para la prevención de transmisión vertical de VIH en gestantes migrantes demandan una perspectiva intercultural, en donde se aborden todas las dimensiones sociales, culturales y de género asociadas a la infección. El entender la realidad local en cuanto a la prevención de transmisión vertical de VIH en población migrante internacional en Chile es esencial para llevar acciones concretas que favorezcan la prevención de transmisión madre-hijo de VIH. En este artículo se presentan algunos conceptos esenciales relacionados a esta temática. También se expone información internacional y nacional sobre riesgos de transmisión vertical de VIH en migrantes gestantes, la importancia del plan nacional de prevención de transmisión vertical de VIH en nuestro país, y algunos esfuerzos que se están realizando para adaptar dicho plan a la realidad de diversidad social y cultural que migrantes gestantes presentan hoy en Chile, como un valioso insumo de salud pública con perspectiva intercultural.
Palabras clave:

Transmisión Vertical de Enfermedad Infecciosa; Migrantes; Atención Prenatal; Servicios Preventivos de Salud 


\begin{abstract}
Preventing vertical transmission of HIV is a challenge for all countries worldwide. The permanent construction of global societies with a variable degree of international migrant population has made it more complex. Health policies, programs, and actions for preventing vertical transmission of HIV in pregnant migrants demand an intercultural perspective, where social, cultural, and gender dimensions associated with the infection are addressed. Understanding the local reality regarding the prevention of vertical transmission in the international migrant population in Chile is essential to carry out concrete actions that favor the prevention of mother-to-child transmission of HIV. This article presents some essential concepts related to this topic. It also presents international and national information on risks of vertical transmission in pregnant migrants, the importance of the national plan for preventing vertical transmission of HIV in our country, and some ongoing efforts to adapt such plan to the reality of social and cultural diversity that pregnant migrants currently present in Chile, as a useful public health instrument with an intercultural perspective.
\end{abstract}

Keywords:

Vertical Transmission of Infectious Disease; Migrants; Prenatal Care; Preventive Health Services

\section{Introducción}

Desde el año 2010 se han asumido diversos compromisos por parte de los Estados Miembros de la Organización Panamericana de la Salud (OPS) con el fin de lograr la eliminación de la transmisión maternoinfantil del virus de inmunodeficiencia humana (VIH) en la Región de las Américas. Los diversos acuerdos y acciones regionales han permitido el logro de importantes avances en la Región, por ejemplo, en América Latina y el Caribe se estima que la tasa de transmisión materno-infantil del VIH disminuyó de $17 \%$ en 2010 a $12 \%$ en 2017 , lo que representa la prevención aproximada de 30.800 infecciones por el VIH en niños y niñas, gracias a diversas intervenciones de eliminación de la transmisión materno-infantil ${ }^{1}$. Estos avances no se han producido de manera uniforme en los distintos países de la región. En el caso de Chile, en los últimos años ha aumentado exponencialmente el número de personas viviendo con $\mathrm{VIH}^{2,3}$. El aumento de casos se produce más en hombres, aun cuando en las mujeres ha aumentado la notificación en etapa de infección por $\mathrm{VIH}^{4}$. Junto con esto, en los últimos años ha aumentado el número de casos notificados correspondientes a personas extranjeras. Estas cifran se dan en un contexto en donde la población migrante internacional corresponde a aproximadamente un $6 \%-7 \%$ del total a nivel país ${ }^{5}$ y está en constante aumento. El año 2017 hubo 3.291 casos notificados de VIH en el sistema de salud público, de los cuales 629 (19\%) correspondieron a migrantes ${ }^{6}$. La situación de exposición de población migrante a enfermedades infecciosas no es particular de Chile. Según datos del 2018 del European Centre for Disease Prevention and Control, el 25\% de los casos de TB que se producen en Europa son diagnosticados en individuos nacidos en países extranjeros ${ }^{7}$. Este escenario mundial, regional y nacional presenta importantes desafíos en materia de salud pública, en prevención, detección y tratamiento, con esfuerzos adicionales en evitar la transmisión vertical desde una mujer embarazada portadora de VIH a su hijo/a.

Es así como, en el contexto de la prevención de la infección por VIH, el manejo del VIH en embarazadas migrantes para la prevención de la trasmisión vertical presenta importantes retos. Los principales desafíos en esta materia dicen relación con la pesquisa para diagnosticar al mayor número de embarazadas que viven con VIH, a la indicación de terapia antirretroviral (TAR) a por lo menos el $90 \%$ de las personas diagnosticadas y al control de la infección mediante carga viral indetectable de por lo menos el $90 \%$ de las embarazadas con TAR para lograr la eliminación de la TV del VIH. De esta forma, se propone una transmisión vertical inferior al $2 \%$, correspondiente a la meta de la OPS. Junto con esto, urge la incorporación efectiva del enfoque de salud intercultural en el abordaje de esta problemática, que permita romper las barreras administrativas, lingüísticas y socioculturales -incluyendo dimensiones de género y socioeconómicas- existentes en la actualidad con algunos grupos migrantes en el acceso y uso del sistema de salud para prevención y tratamiento del VIH y la prevención de su transmisión vertical al que está por nacer. A continuación, se presenta una actualización del escenario país sobre el cómo se aborda esta problemática, y tareas pendientes en la materia para salud pública e infancia.

\section{Migración como determinante social de la salud}

Existe consenso internacional sobre el reconocimiento de la migración como un determinante social de la salud, siendo un proceso dinámico y cambiante que requiere de atención especial en salud poblacional y salud pública. Incluye dimensiones como estilos de vida, condiciones del medio social y comunitario, lo 
socioeconómico, político, cultural y ambiental en la trayectoria de vida de la persona, antes, durante y luego de migrar ${ }^{8}$. En otras palabras, el acto migratorio provoca un punto de inflexión con potenciales cambios profundos y estructurales en múltiples condicionantes sociales que impactan en salud ${ }^{9}$. Estos cambios pueden generar mayores vulnerabilidades y riesgos para la salud de las personas migrantes, de sus familias y de las comunidades que les reciben ${ }^{10}$.

A nivel mundial, se ha observado que la población migrante internacional tiende a ser relativamente joven (en edad reproductiva en su mayoría) ${ }^{9}$ y saludable, o al menos reporta en los primeros años de asentamiento menores problemas de salud que la población local, lo que se conoce como efecto de migrante sano a nivel internacional y también ha sido documentado en Chile $^{11,12}$. Sin embargo, las personas migrantes pueden cambiar su comportamiento y adoptar nuevas prácticas similares a los locales (asimilación), así como perder algunas de sus formas de comprender la realidad o sus costumbres y tradiciones (aculturación). Con esto, se pueden asumir nuevos riesgos para su salud sexual y reproductiva, volverse más vulnerables al alcoholismo y uso de drogas, sufrir cambios alimentarios o verse obligados a permanecer en espacios sin condiciones adecuadas de saneamiento, agua potable, entre otros ${ }^{13}$. También pueden encontrar limitado acceso a servicios de salud básicos, agravado por barreras culturales y lingüísticas que experimentan en países receptores ${ }^{10,14,15}$. Por otra parte, la población migrante puede tener riesgos de enfermedades propias de su país de origen, lo que puede requerir de especial atención en el país receptor migratorio, así como medidas de protección y prevención para la población general ${ }^{16}$. Por tanto, la temática de salud pública y migración internacional es de interés mundial y un permanente desafío global.

\section{Cuidados prenatales en migrantes internacionales}

En Chile no son abundantes los estudios sistemáticos sobre la salud de las madres migrantes, pero se han descrito algunos riesgos sanitarios asociados. Los problemas de salud más frecuentes tanto en personas migrantes como no migrantes son los de tipo obstétrico, $y$ en nuestro medio resulta preocupante que entre las primeras hay una importante proporción sin previsión de salud ${ }^{17,18}$. Otra característica de algunos grupos de embarazadas migrantes es la no asistencia a controles de embarazos o el inicio tardío del cuidado prenatal con la consiguiente pesquisa tardía de la morbilidad materna y fetal, aun cuando más del $80 \%$ de estas gestantes alcanza el mínimo recomendado de tres o más atenciones durante el embarazo ${ }^{17}$. Esto se ha atribuido a la no disponibilidad de la documentación necesaria, desconocimiento del sistema oficial de salud del país, dificultad de acceso físico o financiero, barreras culturales, entre otras ${ }^{19}$.

En el Hospital Clínico San Borja Arriarán, hospital público y el principal de un Servicio de Salud de la Región Metropolitana, donde en el año 2017 más del 60\% de los partos correspondieron a madres extranjeras, en el año 2016 ocurrieron tres muertes maternas, todas de madres migrantes que, según el análisis del Comité de Auditoría de ese establecimiento, no tuvo control del embarazo o este fue tardío ${ }^{18}$. Por otra parte, si se compara a las embarazadas migrantes con las chilenas, se observa que las primeras tienen significativamente mayor riesgo biopsicosocial $(50,1 \% \text { vs } 62,3 \%)^{17}$, entendiendo por tal a las situaciones y condiciones de vida y de trabajo que tienen la capacidad de afectar, en forma negativa, el bienestar y la salud física, psíquica y/o social de la persona ${ }^{13}$.

\section{Población migrante internacional y VIH/SIDA}

La vinculación entre migración y VIH ha sido abordada y estudiada principalmente por organismos internacionales (OPS, OIM, OMS, entre otros), poniendo el acento en que se trata de una relación compleja y multicausal. Por lo mismo, se requieren mayores antecedentes y adecuados diagnósticos de situación para la elaboración de programas y políticas públicas orientadas a este grupo. A lo largo del proceso migratorio, en algunos casos las personas migran con la condición desde el país de origen, en otros casos el país de destino puede ser el lugar de mayor contagio y, en otros, el trayecto es la instancia de mayor riesgo ${ }^{10,20,21}$.

En Chile hay dos enfermedades infecciosas que son de mayor preocupación por su más alta frecuencia en los países de origen de algunos grupos de migrantes: la infección con VIH y la tuberculosis (TBC) $)^{13}$. A nivel nacional el número de personas contagiadas con VIH subió de 2.982 casos en 2010 a 6.948 casos en 2018. En la población extranjera esta cifra se incrementó para los mismos años de 41 casos detectados en 2010 a 2.580 en 2018. En 2015, para este mismo grupo poblacional, el porcentaje de personas viviendo con VIH representaba el 9,19\% del total de personas contagiadas, mientras que en 2018 representa el $37,13 \%{ }^{6}$. En esta misma línea, el informe epidemiológico del Departamento de Enfermedades Infecciosas del Ministerio de Salud, al 28 de noviembre de $2017^{6}$ evidenciaba el incremento de los casos de VIH en el país en un 96\% entre el periodo 2010 y 2017. Del total de casos detectados entre enero-junio de 2018 un 36\% correspondía a extranjeros. Al desglosar ese grupo según nacionalidades se muestra que el $36,4 \%$ de dichos casos correspondía a 
Tabla 1. Indicadores de salud (VIH) en Chile y países de mayor ingreso de migrantes a Chile

\begin{tabular}{|c|c|c|c|c|c|c|c|c|c|}
\hline \multicolumn{2}{|l|}{ Indicador } & Chile & Argent & Bolivia & Colombia & Ecuador & Haití & Peru & Venez \\
\hline \multicolumn{2}{|l|}{ Ingreso PIB (PPA)* } & 26.905 & 21.528 & 7.900 & 15.055 & 11.864 & 1.870 & 13.993 & 10.399 \\
\hline $\begin{array}{l}\text { Mortalidad infantil por } \\
1.000 \text { nacidos vivos }\end{array}$ & $\begin{array}{l}<1 \text { año } \\
<5 \text { años }\end{array}$ & $\begin{array}{c}7 \\
8,3\end{array}$ & $\begin{array}{l}10 \\
11\end{array}$ & $\begin{array}{c}35 \\
36,9\end{array}$ & $\begin{array}{c}14 \\
15,3\end{array}$ & $\begin{array}{c}16 \\
20,9\end{array}$ & $\begin{array}{c}48,2 \\
67\end{array}$ & $\begin{array}{l}18 \\
15\end{array}$ & $\begin{array}{c}12 \\
16,3\end{array}$ \\
\hline \multicolumn{2}{|c|}{ Mortalidad materna por 100.000 nacidos vivos } & 22 & 52 & 206 & 64 & 64 & 359 & 68 & 95 \\
\hline \multicolumn{2}{|c|}{$\begin{array}{l}\text { Incidencia VIH (casos nuevos anuales x } 100.000 \\
\text { habitantes) }\end{array}$} & 0,28 & 0,13 & 0,10 & 0,12 & 0,12 & 0,77 & 0,09 & 0,21 \\
\hline \multicolumn{2}{|c|}{ Prevalencia VIH por 100 adultos } & 0,34 & 0,27 & 0,17 & 0,25 & 0,20 & 1,38 & 0,22 & 0,38 \\
\hline
\end{tabular}

Fuente: Armas, Cabieses, Wolff et al. 2018. Salud y proceso migratorio. Anales de Instituto de Chile. PIB: Producto Interno Bruto; PPA: Paridad de Poder Adquisitivo.

personas provenientes de Venezuela; el 28,6\% a personas de Haití; y el 11,2\%, de Colombia. Este porcentaje, sin duda, refleja una sobrerrepresentación de extranjeros $^{2}$. Es importante observar estos datos de acuerdo con las prevalencias e incidencias de esta enfermedad en los países de origen, lo que se presenta en la tabla $1^{21}$. Como se puede observar, muchos de los países de mayor ingreso a Chile por parte de extranjeros presentan tasas de infección por VIH más elevadas que en nuestro país.

Datos adicionales ofrece la base de egresos hospitalarios de Chile ${ }^{22}$. Para el 2015, se observa mayor concentración de migrantes con enfermedades infecciosas en las regiones Metropolitana, Arica y Parinacota, y Antofagasta, equivalente a las de mayor densidad de migrantes en el país (tabla 2). Comparado con egresos hospitalarios en chilenos el año 2015, en población migrante se observa mayor proporción de registros de VIH, TBC y algunas enfermedades infecciosas de la piel (tabla 3). Esta información es representativa de todo el territorio nacional que accede al sistema de salud, pero tiene fallas de sesgo de selección al poder existir grupos de migrantes en algún riesgo que, por desconocimiento o temor, no acceden al sistema de salud, así como posibles errores de registro de estatus migratorio o de nacionalidad, que deben seguirse revisando y actualizando.

El aumento de la incidencia y mortalidad asociada al VIH en Chile no es atribuible exclusivamente a la población migrante internacional. Se observa un aumento importante del VIH en los últimos años en jóvenes y hombres chilenos, grupo en el cual ha disminuido el uso de condón ${ }^{23}$, principal medida preventiva reconocida por este grupo. Informes recientes ministeriales sobre enfermedades infectocontagiosas indican también el aumento de estas condiciones en personas con adicciones, personas privadas de libertad y en situación de calle ${ }^{24}$. Sin embargo, dada la sobrerrepresentación relativa en el grupo de migrantes con VIH
Tabla 2. Egresos hospitalarios 2015, migrantes internacionales y chilenos, capítulo enfermedades infecciosas

\begin{tabular}{|c|c|c|c|c|}
\hline Sexo & \multicolumn{2}{|c|}{$\begin{array}{c}\text { Chileno } \\
(n=37.521)\end{array}$} & \multicolumn{2}{|c|}{$\begin{array}{l}\text { Inmigrante } \\
(n=264)\end{array}$} \\
\hline Hombre & $53,9 \%$ & $(20.206)$ & $60,2 \%$ & (159) \\
\hline Mujer & $46,1 \%$ & $(17.313)$ & $39,8 \%$ & (105) \\
\hline \multicolumn{5}{|l|}{ Edad } \\
\hline$<14$ & $38,5 \%$ & $(14.453)$ & $17,4 \%$ & (46) \\
\hline $15-29$ & $12,5 \%$ & (4.683) & $23,9 \%$ & (63) \\
\hline $30-44$ & $11,5 \%$ & (4.309) & $29,9 \%$ & (79) \\
\hline $45-64$ & $15,7 \%$ & (5.873) & $16,7 \%$ & (44) \\
\hline$>65$ & $21,9 \%$ & (8.203) & $12,1 \%$ & (32) \\
\hline \multicolumn{5}{|l|}{ Previsión de salud } \\
\hline FONASA & $71,9 \%$ & $(26.973)$ & $42,8 \%$ & (113) \\
\hline ISAPRE & $20,8 \%$ & (7.795) & $28,0 \%$ & (74) \\
\hline Sin previsión & $1,8 \%$ & $(675)$ & $22,7 \%$ & (60) \\
\hline Otros & $5,5 \%$ & (2.078) & $6,4 \%$ & (17) \\
\hline \multicolumn{5}{|l|}{ Región del país } \\
\hline Tarapacá & $1,7 \%$ & (628) & $8,7 \%$ & (23) \\
\hline Antofagasta & $3,6 \%$ & $(1.340)$ & $12,9 \%$ & (34) \\
\hline Atacama & $1,1 \%$ & $(401)$ & $0,0 \%$ & (0) \\
\hline Coquimbo & $2,5 \%$ & (951) & $0,8 \%$ & (2) \\
\hline Valparaíso & $11,4 \%$ & $(4.296)$ & $1,9 \%$ & (5) \\
\hline Libertador B. O’Higgins & $4,2 \%$ & $(1.564)$ & $2,7 \%$ & (7) \\
\hline Maule & $5,1 \%$ & (1.926) & $1,1 \%$ & (3) \\
\hline Bíobío & $13,9 \%$ & $(5.197)$ & $0,4 \%$ & (1) \\
\hline La Araucanía & $6,5 \%$ & $(2.447)$ & $1,1 \%$ & (3) \\
\hline Los Lagos & $5,7 \%$ & $(2.141)$ & $0,8 \%$ & (2) \\
\hline $\begin{array}{l}\text { Aisén del Gral. C. Ibáñez } \\
\text { del Campo }\end{array}$ & $0,8 \%$ & $(298)$ & $0,0 \%$ & (0) \\
\hline $\begin{array}{l}\text { Magallanes y de La Antártica } \\
\text { Chilena }\end{array}$ & $0,9 \%$ & (330) & $0,0 \%$ & (0) \\
\hline Metropolitana de Santiago & $36,9 \%$ & $(13.836)$ & $62,1 \%$ & $(164)$ \\
\hline Los Ríos & $4,1 \%$ & $(1.546)$ & $1,1 \%$ & (3) \\
\hline Arica y Parinacota & $1,3 \%$ & $(502)$ & $2,7 \%$ & (7) \\
\hline Sin Reportar & $0,3 \%$ & $(118)$ & $0,0 \%$ & (0) \\
\hline
\end{tabular}

Fuente: elaboración propia. 
Tabla 3. Egresos hospitalarios 2015 en Chile, migrantes internacionales y chilenos, tipo de patología capítulo de enfermedades infecciosas

\begin{tabular}{|c|c|c|c|c|}
\hline \multirow[b]{2}{*}{ Enfermedades infecciosas intestinales } & \multicolumn{2}{|c|}{$\begin{array}{c}\text { Chileno } \\
(n=37.521)\end{array}$} & \multicolumn{2}{|c|}{$\begin{array}{l}\text { Inmigrante } \\
(n=264)\end{array}$} \\
\hline & $47,1 \%$ & $(17.666)$ & $38,6 \%$ & $(102)$ \\
\hline Tuberculosis & $3,8 \%$ & $(1.435)$ & $13,6 \%$ & (36) \\
\hline Ciertas zoonosis bacterianas & $0,2 \%$ & $(60)$ & $0,0 \%$ & (0) \\
\hline Otras enfermedades bacterianas & $21,3 \%$ & (8.009) & $14,0 \%$ & (37) \\
\hline Infecciones con modo de transmisión predominantemente sexual & $3,0 \%$ & $(1.111)$ & $3,8 \%$ & (10) \\
\hline Infecciones virales del sistema nervioso central & $3,9 \%$ & $(1.466)$ & $1,9 \%$ & (5) \\
\hline Fiebres virales transmitidas por artrópodos y fiebres virales hemorrágicas & $0,0 \%$ & $(16)$ & $0,8 \%$ & $(2)$ \\
\hline Infecciones virales caracterizadas por lesiones de la piel y de las membranas mucosas & $5,5 \%$ & $(2.060)$ & $4,5 \%$ & $(12)$ \\
\hline Hepatitis viral & $2,2 \%$ & $(837)$ & $3,0 \%$ & (8) \\
\hline Enfermedad por virus de la inmunodeficiencia humana [VIH] & $4,5 \%$ & $(1.680)$ & $14,4 \%$ & (38) \\
\hline Otras enfermedades virales & $4,7 \%$ & $(1.774)$ & $3,8 \%$ & (10) \\
\hline Enfermedades debidas a protozoarios & $0,3 \%$ & $(107)$ & $0,8 \%$ & (2) \\
\hline Helmintiasis & $2,0 \%$ & $(753)$ & $0,4 \%$ & (1) \\
\hline Pediculosis, acariasis y otras infestaciones & $0,2 \%$ & $(71)$ & $0,4 \%$ & (1) \\
\hline
\end{tabular}

Fuente: elaboración propia.

en relación con su densidad poblacional en el país, sin duda son un grupo relevante en materia de salud pública y de investigación. Más aún cuando el foco se pone en migrantes gestantes y los riesgos del que está por nacer en contextos donde pueden existir menor cantidad de adaptaciones de protocolos de transmisión vertical disponibles a la luz de necesidades de grupos poblacionales específicos.

\section{Factores de riesgo de gestantes para adquirir VIH y trasmitirlo a sus recién nacidos}

Es de especial preocupación el grupo de mujeres migrantes jóvenes en plena edad reproductiva, ya que la evidencia mundial sugiere que frecuentemente las mujeres migrantes buscan apoyo médico en estados avanzados de gravidez, siendo la mortalidad materna mayor en ellas en comparación a las mujeres nati$\operatorname{vas}^{17,20,25}$. Esto genera una alerta en salud materno-fetal $\mathrm{y}$ en particular con respecto a enfermedades transmisibles al recién nacido ${ }^{26,27}$, ya que enfermedades como VIH, hepatitis B, HTLV1 y ciertas parasitosis, para las cuales un diagnóstico precoz y tratamiento oportuno son fundamentales para el pronóstico de la madre y para prevenir su transmisión al recién nacido, son muchas veces mayores en sus regiones de origen.

A nivel regional, en el 2015 se estimó que alrededor de 670.000 mujeres de 15 años de edad o mayo- res tenían la infección por el VIH. De ellas, cerca del $58 \%$ estaban en tratamiento antirretroviral (TAR) ${ }^{28,29}$. La cobertura del TAR en las embarazadas aumentó de un $55 \%$ en 2010 a un $88 \%$ en 2015 y se estimó que la tasa de transmisión materno-infantil se redujo de un $15 \%$ en 2010 a un $8 \%$ en 2015 . El número de infecciones nuevas por el VIH en los niños (de 0 a 14 años de edad) descendió en un 55\% entre el 2010 y el 2015 -de 4.700 (3.500-6.400) en el 2010 a 2100 (1600-2900) en el 2015-, y en el mismo periodo se previnieron cerca de 28.000 infecciones nuevas por el VIH. El tamizaje de la sífilis en las embarazadas que acudieron a la atención prenatal en la región disminuyó de 74\% en 2011 a 69\% en 2017. La tasa de incidencia de la sífilis congénita en América Latina y el Caribe, por su parte, ha aumentado desde 2010 y alcanzó 2,1 casos por 1.000 nacidos vivos y más de 28.800 casos notificados en $2017^{1}$.

Para el caso de Chile, datos actualizados de incidencias y prevalencias de estas infecciones en gestantes migrantes no están siempre disponibles; sin embargo, un estudio del Complejo Asistencial Barros Luco indicó una incidencia de hepatitis B de 2,4\% al total de recién nacidos en un periodo de 12 meses (julio de 2017 a junio de 2018) ( $\mathrm{n}=35$ casos, de los cuales 34 correspondían a migrantes $)^{27}$. Además, en Chile el Programa Nacional de Inmunizaciones (PNI) alcanza coberturas superiores al $90 \%$ desde los años 80 , mientras que otros países latinoamericanos han tenido históricamente coberturas inferiores o muy variables en vacunas, y muy 
probablemente parte de la población adulta migrante sea aún susceptible a infecciones inmunoprevenibles, y por tanto foco de posibles brotes de infecciones transmisibles como el sarampión o la rubeola ${ }^{30}$.

La evidencia internacional indica que existen múltiples factores asociados o predisponentes al contagio vertical de VIH por parte de mujeres migrantes hacia sus hijos. Una búsqueda de revisiones sistemáticas desarrollada por las autoras en PubMed (08 de febrero de 2019) arrojó 18 artículos relevantes, la mayoría de carácter clínico y algunos de interés más contextual o salud pública. Todos ellos dan cuenta de la complejidad de dimensiones implicadas en la transmisión de VIH por parte de mujeres gestantes a sus hijos. Haciendo uso de esta evidencia, y de evidencia más amplia de factores asociados a contagio de VIH en población femenina mundial, se reconocen una serie de dimensiones importantes de abordar. En primer lugar, están las dimensiones relacionadas a factores demográficos generales y a la etapa del ciclo vital en la que se encuentran y que pueden ser comunes a otras mujeres no migrantes (edad joven y etapa reproductiva con búsqueda de embarazo; así como factores estructurales como estado civil y pertenencia étnica) ${ }^{31}$. En segundo lugar, están aquellos factores socioeconómicos o de vulnerabilidad social de mujeres migrantes, como son condiciones educacionales, de ingreso del hogar y de trabajo a las que son sometidas cuando migran y que pueden ponerlas en riesgo ${ }^{31,32}$. Esto se entrelaza con las dimensiones de desigualdad social y de género, que impactan en forma severa en salud en el caso de vivir en condiciones de gran desventaja como pobreza, o en contextos familiares o sociales de represión como machismo o abuso basado en género. Casos especiales de lo anterior son migrantes trabajadoras sexuales o sometidas a refugio/ asilo, a violencia o conflicto, a trata o tráfico de personas, que las expone a un mayor riesgo de adquirir enfermedades incluyendo $\mathrm{VIH}^{19}$. En tercer lugar, existen factores asociados al sistema de salud del país receptor y a las politicas de protección social de ese país, incluyendo el beneficio de cobertura en salud para problemas como el VIH en mujeres migrantes y sus hijos por nacer. Sistemas de salud cerrados, que excluyen a personas migrantes de cualquier tipo (o grupos específicos como aquellos en situación irregular) corren el riesgo de postergar medidas preventivas y diagnósticas oportunas, y a largo plazo deber costear problemas de salud de mayor urgencia y severidad en estos grupos poblacionales, tal y como lo han documentado estudios de otros países como Alemania ${ }^{33}$. La inexistencia de un protocolo de prevención de transmisión vertical de VIH en países receptores es sin duda un factor de riesgo para su transmisión en el que está por nacer. En cuarto lugar, existen factores asociados al proceso migratorio y los potenciales riesgos de contagio o de mayor transmisión vertical, tales como condiciones de vida antes de migrar, riesgos asociados al acto migratorio en sí mismo (trata, abuso, violencia sexual, entre otros), riesgos asociados al proceso de llegada inicial al nuevo país (soledad, aislamiento, exclusión, pobreza y hacinamiento $)^{34}$. En quinto lugar, se pueden observar factores asociados a la enfermedad y sus condicionantes específicas, tales como número de parejas sexuales, actitudes y conductas hacia el VIH, conocimiento sobre la enfermedad y sus riesgos personales, entre otros ${ }^{35}$. También resultan importante condicionantes asociadas como consumo de sustancias, de alcohol, situación de calle, maltrato en la pareja y abuso sexual. El detalle de revisiones sistemáticas analizadas apara esta sección aparecen en la tabla 4.

\section{Prevención vertical de VIH en Chile}

Dado que el manejo adecuado de las gestantes viviendo con VIH (VVIH) ha demostrado, según la evidencia científica, ser la intervención más costo-efectiva para evitar la transmisión de esta patología a los recién nacidos, la legislación chilena ha considerado la detección y el tratamiento oportuno para ambas infecciones. En VIH, el primer protocolo de prevención de la transmisión vertical (ACTG 076) en Chile data del año $1996^{36}$. La Norma de Prevención de la Transmisión Vertical del VIH, del año 2005, incluye el ofrecimiento universal del test a las embarazadas, protocolo farmacológico a las gestantes VVIH y sus hijos/as (garantizado en GES), y sucedáneos de leche materna durante seis meses ${ }^{37}$. La detección de VIH en las gestantes está considerada en el Examen de Medicina Preventiva de la Ley GES. Las mujeres migrantes embarazadas por su parte cuentan con atención prenatal completa sin costo alguno en el sistema público de atención en Chile desde 2006, que incluye la detección de VIH y el cuidado del que está por nacer ${ }^{38}$. La norma además incluye el protocolo diagnóstico (exámenes de PCR al nacer, al mes y 3 meses de vida) del RN, estudio inmunológico (CD4) y seguimiento del RN hasta los 18 meses. Es decir, entrega una atención integral al binomio madrehijo. Es una prestación asegurada en su totalidad por GES para todas las embarazadas, incluidas las inmigrantes.

De acuerdo con el informe Ministerial de transmisión vertical de VIH de $2012^{38}$, el diagnóstico de VIH en la embarazada y la aplicación del protocolo completo para la prevención de transmisión vertical permiten reducir la tasa de transmisión de entre $13 \%$ a $48 \%$ hasta menos de $2 \%$. La transmisión vertical del $\mathrm{VIH}$, se produce en un 35\% de los casos durante el embarazo y aproximadamente en un 65\% durante el parto, por exposición del recién nacido a sangre materna, secreciones cervicovaginales o líquido amniótico. 


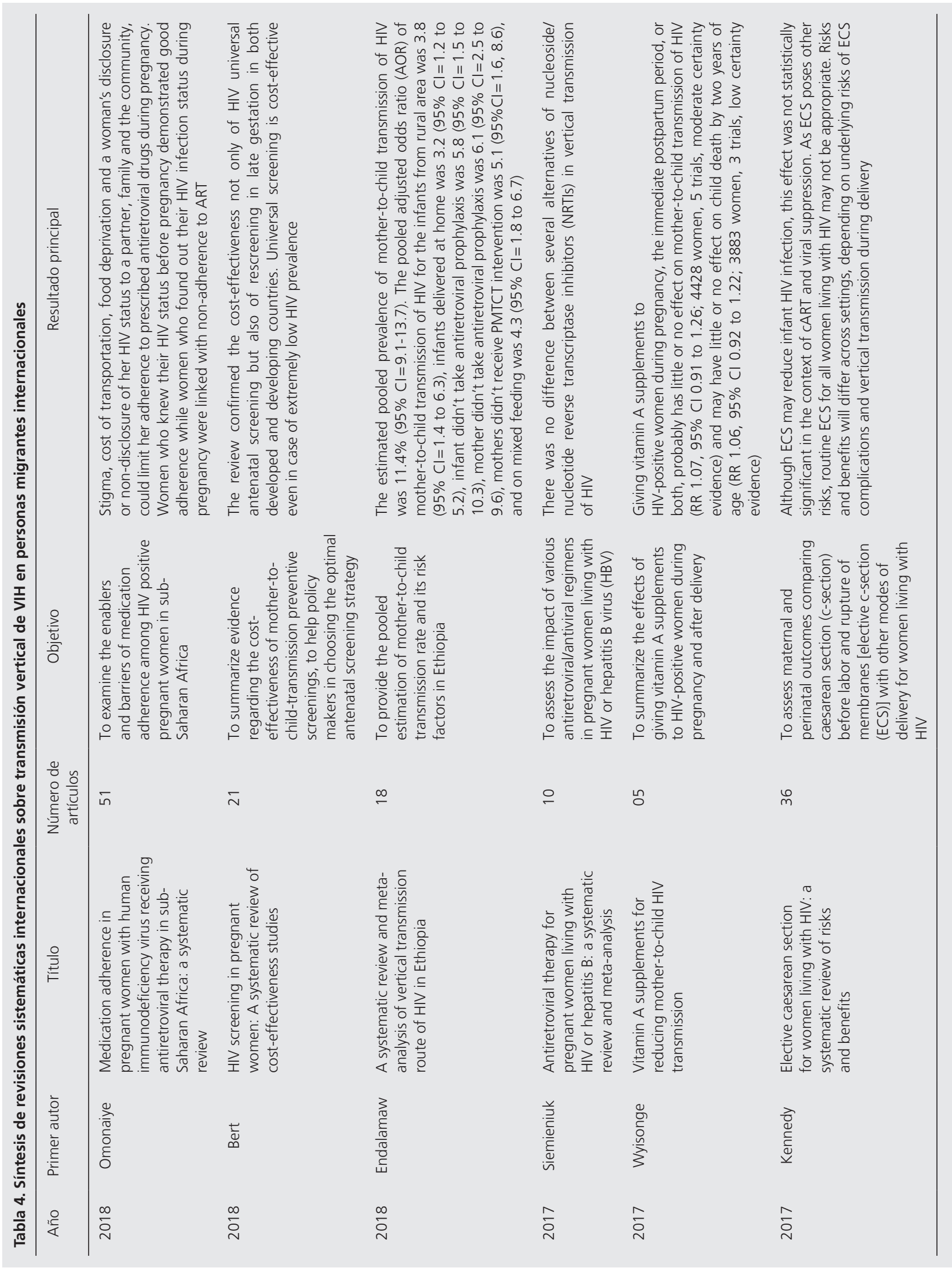




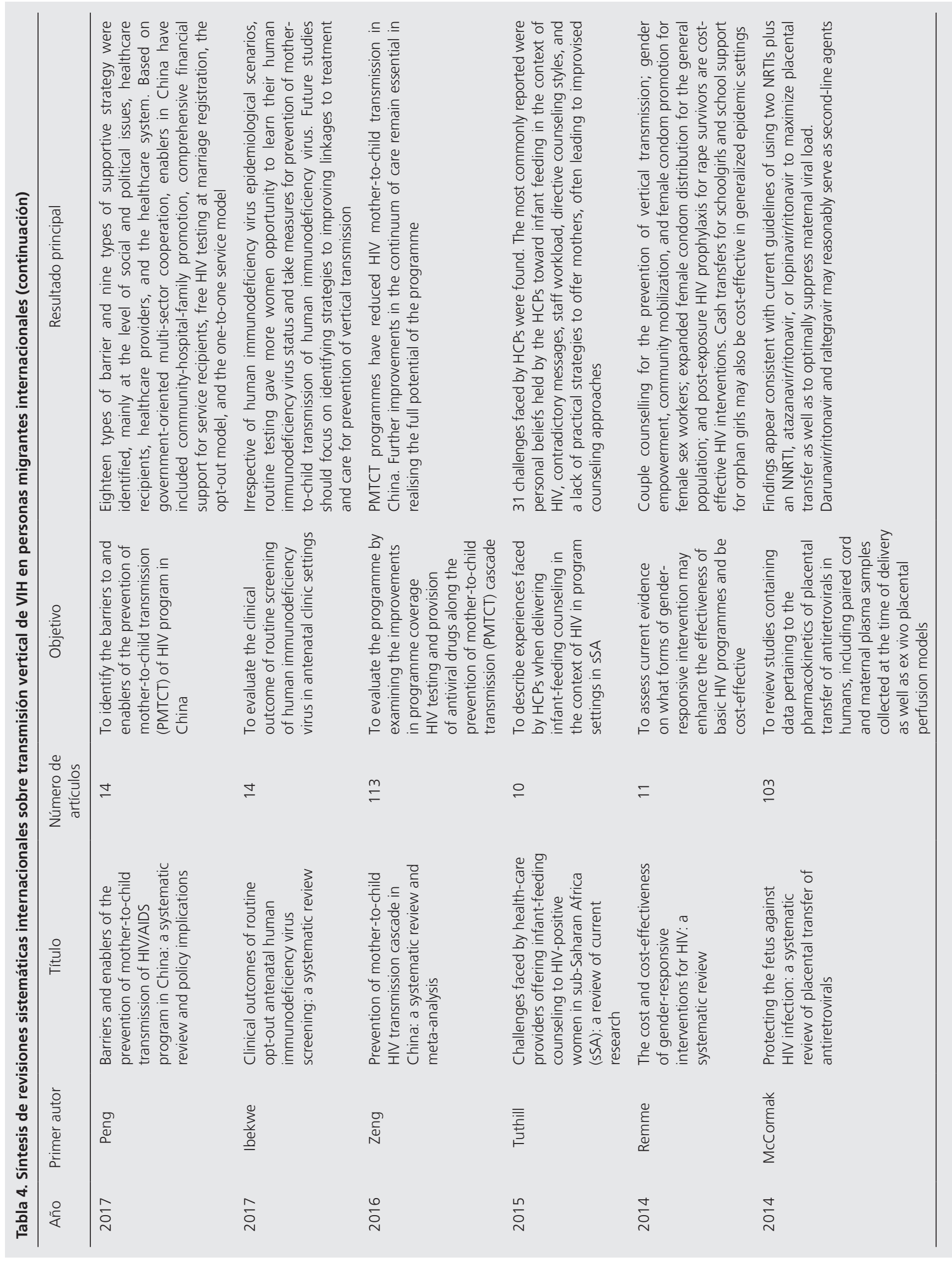




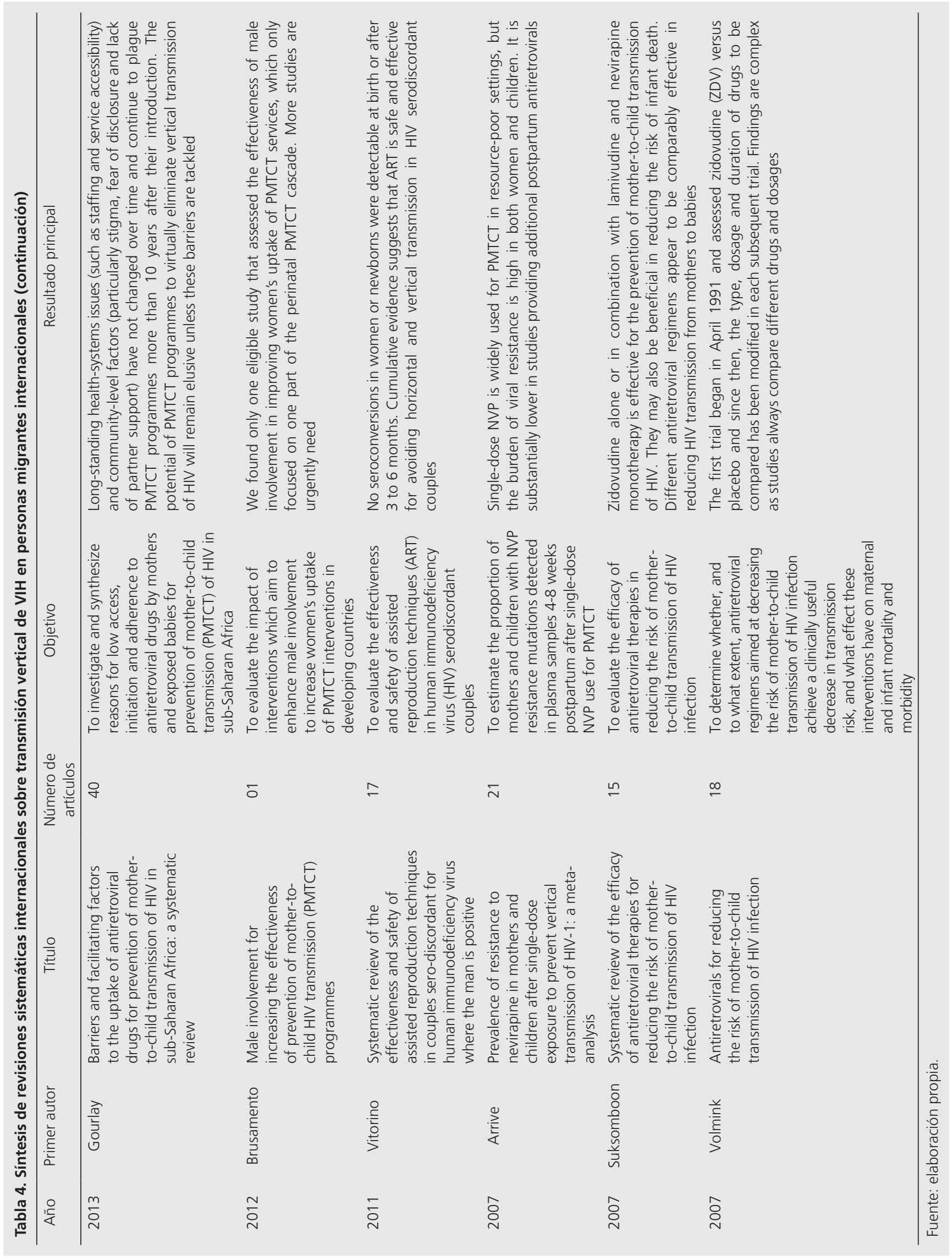


La lactancia materna agrega un riesgo adicional de 14\% hasta $29 \%$. Datos nacionales dan cuenta de una reducción importante de las tasas de transmisión vertical en los últimos cinco años, de 5,1\% el 2014 (12/232) a $2,4 \%$ el $2018(9 / 374)^{39}$. Estas mejoras de prevención de transmisión vertical de VIH han venido instalándose gracias a esfuerzos ministeriales desde los años $90 \mathrm{~s}^{40}$.

El objetivo principal del protocolo ministerial de prevención de transmisión vertical de VIH es reducir la transmisión materno-infantil del VIH y la sífilis en los niños y niñas expuestos/as al riesgo. Para lograr este objetivo se perfila como necesario lograr diagnosticar el mayor número de embarazadas; que la totalidad de las embarazadas VVIH reciban TAR altamente efectiva; que alcancen la indetectabilidad en las últimas cuatro semanas de gestación o cercano al parto; que la vía de parto y el manejo inmediato del RN aseguren el menor riesgo de TV; y prohibir lactancia materna asegurando la lactancia artificial. En el marco de dicho objetivo las metas ministeriales son: i) Disminuir la transmisión vertical del VIH al 2\% o menos; y ii) Mantener o disminuir la incidencia de la sífilis congénita, incluidos los mortinatos, a 0,5 casos por 1.000 nacidos vivos. Este protocolo incluye consideraciones para las distintas etapas involucradas en la transmisión vertical de VIH: embarazo, parto, puerperio, lactancia y cuidado de niños al menos hasta los 18 meses. En cada una de estas etapas se establecen pasos a seguir, según el caso de desconocimiento del diagnóstico, sospecha diagnóstica y diagnóstico definido.

El Ministerio de Salud de Chile, como máxima autoridad sanitaria, ha atendido la necesidad de velar por la protección en salud de personas migrantes que hoy residen en nuestro territorio. Para ello, desde el año 2006 a la fecha se han ido creando normativas técnicas y decretos que han aumentado el alcance de la cobertura en salud para este grupo, hasta su cúlmine con el lanzamiento de la Política de Salud de Migrantes del año $2017^{41}$, que hoy se encuentra en desarrollo de su Plan de Acción. Este plan de acción incluye metas, indicadores y actividades específicas pertinentes para el cuidado de gestantes y niños migrantes, así como el manejo de enfermedades de notificación obligatoria, de las cuales VIH se ha considerado como una trazadora principal.

En Chile aún no se han hecho estudios que permitan comprender en profundidad la situación de riesgo de mujeres migrantes embarazadas que viven con VIH, ni sus conocimientos o prácticas asociadas a la transmisión vertical. Por ello, surge como desafío importante desarrollar investigaciones de primer nivel que documenten en forma seria y sistemática la compleja relación entre migración internacional, embarazo y transmisión vertical de VIH, desde la perspectiva de inteligencia en salud pública y de cara a los protoco- los actuales que existen en esta materia en la actualidad en el país. Se recomiendan estudios cuantitativos que identifiquen y analicen patrones de prevalencia, riesgos y complicaciones asociadas, así como también de carácter cualitativo que permitan aproximarnos a las creencias, experiencias y necesidades específicas de migrantes gestantes VVIH y de equipos de salud en nuestro país. De esta manera podremos identificar las brechas que hoy existen entre lo que estas poblaciones necesitan y lo que actualmente el sistema de salud tiene dispuesto. Solo así se podrán implementar mejoras sólidas y basadas en la evidencia que aporten a la prevención efectiva y segura de transmisión vertical de VIH en todo niño/a que está por nacer en nuestro país, así como un adecuado acompañamiento, sin estigmatización ni discriminación alguna, para aquellos casos en los que el sistema haya llegado tarde en estas mujeres y niños/as, desde un enfoque intercultural de protección y humanización del cuidado de nuestro sistema de salud público formal ${ }^{42}$.

\section{Experiencias nacionales de acercamiento del protocolo nacional de prevención de TV a mi- grantes gestantes}

En reconocimiento del complejo contexto actual, es importante describir algunas de las valiosas experiencias y esfuerzos que se están desarrollando en Chile para acercar o adaptar el protocolo nacional de prevención de transmisión vertical de VIH en gestantes migrantes $^{43}$. A nivel central, el Ministerio de Salud ha resguardado y ofertado la aplicación de la normativa de prevención de transmisión vertical a toda la población beneficiaria, sin excepciones de ningún tipo ${ }^{38}$. Para cada caso particular, se coordina en red el seguimiento y se activa el trabajo de comités locales y regionales, generando acciones de mejora cuando es pertinente, como puede serlo el caso de gestantes migrantes. Hay esfuerzos de entrenamiento en migración y salud a equipos de salud de nivel primario y secundario, adaptaciones idiomáticas en folletería informativa y diversas comunas con alta densidad de migrantes cuentan con facilitador lingüístico y mediador intercultural, en especial para el caso de migrantes gestantes de origen haitiano ${ }^{44}$. Respecto de experiencias locales, se destaca el caso del Complejo Asistencial Dr. Sótero del Río (CASR), donde se han llevado a cabo diversas acciones con el fin de asegurar la adherencia al protocolo de prevención de TV HIV por parte de madres haitianas ${ }^{45}$.

\section{Conclusión}

Chile cuenta con excelentes indicadores de salud materno-infantil. Ello se ha logrado después de un 
gran esfuerzo que se ha mantenido durante muchos años y alcanzado ya a varias generaciones de personas en las que se ha creado una cultura de los cuidados médicos en el embarazo y en la infancia. Chile hoy se enfrenta al desafío de acercar a las mujeres migrantes al sistema de salud formal, para que conozcan y valoren los beneficios que nuestro sistema ofrece desde una perspectiva humana y competente interculturalmente, que considere dimensiones socioeconómicas, culturales y de género vinculadas al proceso vital que están experimentando.

Desde este enfoque Chile tiene la tarea de resolver cómo prevenir la transmisión vertical de VIH en mujeres con diferentes, saberes, expectativas y conocimientos respecto de los procesos de salud/enfermedad. Para eso es necesario que el sistema de salud amplíe su visión biomédica particular, hacia formas diversas de entender y enfrentar la enfermedad. Interesantes esfuerzos se están desarrollando en esta línea en Chile en los últimos años, los que merecen mayor atención y apoyo. Nueva investigación cuantitativa y cualitativa dedicada a esta temática podría ser crítica para acercarnos a necesidades particulares de gestantes migrantes VVIH, así como a posibles mejoras y adaptaciones que nuestro sistema de salud puede requerir para acortar las brechas de uso efectivo del protocolo nacional de prevención de transmisión vertical en esta población.

\section{Conflicto de intereses}

Las autoras declaran no tener conflictos de intereses.

\section{Referencias}

1. Organización Panamericana de la Salud. Nuevas generaciones sin la infección por el VIH, la sífilis, la hepatitis B y la enfermedad de Chagas en las Américas 2018. Whashignton, D.C.; 2019.

2. Ministerio de Salud de Chile. Ministerio de Salud: "Durante todo el 2018 se registraron 6.948 nuevos casos de VIH en Chile". 14 de febrero de 2019.

3. Goldstein E. Evolución de VIH/SIDA en Chile y países seleccionados de América Latina. Asesoría Técnica Parlamentaria. 2019.

4. Ministerio de Salud. Situación epidemiológica de las infecciones de trasmisión sexual. 2016.

5. Instituto Nacional de Estadisticas, Departamento de Extranjería y Migración. Estimación de Personas Extranjeras Residentes en Chile. 31 de diciembre de 2018. 2019. p. 24.

6. Ministerio de Salud de Chile. Informe Nacional: Evolución de infección VIH/ SIDA Chile 1984-2012. Rev Chil Infect. 20117;32(1):17-43.

7. Sánchez-Montalvá A, Salvador F, MolinaMorant D, Molina I. Tuberculosis and immigration. Enferm Infecc Microbiol Clin. 2018 Aug 1;36(7):446-55.

8. Cabieses B, Bernales M, McIntyre AM. La migración internacional como determinante social de la salud en Chile: evidencia y propuestas para políticas públicas. Universida. Santiago: Universidad del Desarrollo; 2017.

9. Cabieses B. Investigación en migración y salud en Chile: Avanzando. Rev Med Chile. 2016 Aug 1;144(8):1093-4.

10. Van der Laat C. La migración como determinante social de la salud. In: Cabieses B, Bernales M, McIntyre AM, editors. La migración internacional como determinante social de la salud en Chile: evidencia y propuestas para políticas públicas. Santiago; 2017. p. 29-38.

11. Helgesson M, Johansson B, Nordquist T, Vingård E, Svartengren M. Healthy migrant effect in the Swedish context: A register-based, longitudinal cohort study. BMJ Open. 2019 Mar 1;9(3).

12. Cabieses BB, Tunstall H, Pickett K. Testing the Latino Paradox in Latin America: A Population-Based Study of Intra-regional Immigrants in Chile. Rev Med Chile [Internet]. 2013 [cited 2020 Jun 12];141(10):1255-65. Available from: https://pubmed.ncbi.nlm.nih. gov/24522353/.

13. Colegio Médico de Chile, Instituto de Ciencias e Innovación en Medicina (ICIM) Facultad de Medicina Clínica Alemana Universidad del Desarrollo, Sociedad Chilena de Infectología. Hacia una comprensión integral de la relación entre migración internacional y enfermedades infecciosas. De la creencia a la evidencia para la acción sanitaria en Chile. Cabieses B, Libuy M, Dabanch J, editors. Santiago de Chile; 2019. 125 p.

14. United Nations. Trends in total migrant stock: the 2005 revision. Washington, DC; 2006.

15. World Health Organization. Migration and health: key issues Migration and Health.

16. Organización Internacional para las Migraciones. Migración Saludable en América Central. 2012;

17. Bustos Costa P. Relación entre Estatus Migratorio y Resultados de Embarazo y Parto. Análisis en Embarazadas que se Controlaron en Establecimientos de la Red Municipal de Atención Primaria de Salud en la Comuna de Recoleta en el año 2012. Universidad de Chile; 2012.

18. Ministerio de Salud C, Servicio de Salud Metropolitano Central, Servicio de Pediatría. Informes de Auditoría Materno Infantil. Hospital Clínico San Borja Arriarán 2016-2017. 2017.

19. Peng Z, Wang S, Xu B, Wang W. Barriers and enablers of the prevention of motherto-child transmission of HIV/AIDS program in China: a systematic review and policy implications. Int J Infect Dis. 2017;55:72-80.

20. Cortés Castellanos P. Mujeres migrantes de América Latina y el Caribe: derechos humanos, mitos y duras realidades. CEPAL-SER. Santiago de Chile 4: Programa Regional de Población y Desarrollo Centro Latinoamericano y Caribeño de Demografía (CELADE)División de Población/Fondo de Población de las Naciones Unidas; 2005. $83 \mathrm{p}$.

21. Armas R, Cabieses B, Wolff M, Norero C, Rodríguez J, Reyes H. Salud y proceso migratorio actual en Chile. An del Inst Chile. 2018;37:131-62.

22. Departamento de Estadísticas e Información de Salud. Ministerio de Salud. Egresos hospitalarios. 2019.

23. Castro-Sandoval G, Carrasco-Portiño M, Solar-Bustos F, Catrien-Carrillo M, Garcés-González C, MarticorenaGuajardo C. Impacto de las políticas de educación sexual en la salud sexual y reproductiva adolescente en el sur de Chile, período 2010-2017. Rev Chil Obs Ginecol. 2019;84(1):28-40.

24. Ministerio de Salud de Chile. INFORME DE SITUACIÓN EPIDEMIOLÓGICA Y OPERACIONAL DEL PROGRAMA NACIONAL DE TUBERCULOSIS 2017. Santiago de Chile; 2018. 
25. Rojas N, Silva C. La Migración en Chile: Breve reporte y caracterización. Observatorio Iberoamericano sobre Movilidad Humana, Migraciones y Desarrollo. 2016.

26. Izquierdo Copiz G, Reyes A, Delpiano $\mathrm{L}$, et al. Inmigración e impacto en la infectología de recién nacidos. Rev Chil Infectol. 2017;34(4):374-6.

27. Izquierdo G, Bustos S, González Á, et al. Cribado de virus de hepatitis $B$ en mujeres embarazadas: inmigrantes, $y$ chilenas con conductas de riesgo. Manejo del binomio madre-hijo: Plan piloto. Rev Chil Infectología [Internet]. 2019 [cited 2020 Jun 12];36(5):576-84. Available from: www.revinf.cl.

28. Joint United Nations Programme on HIV/AIDS. Programme on HIV/AIDS Prevention Gap Report [Internet]. Geneva; 2016 [cited 2020 Jun 12]. Available from: https://www.unaids. org/en/resources/documents/2016/ prevention-gap.

29. OPS. ETMI-PLUS: marco para la eliminación de la transmisión maternoinfantil del VIH, la sífilis, la hepatitis y la enfermedad de Chagas [Internet]. 2017. Available from: https://www.paho.org/hq/ dmdocuments/2017/2017-cha-etmi-plusmarco-vih-hep-chagas.pdf.

30. Alarcón Y, Balcells ME. Enfermedades infecciosas y migración. Una responsabilidad compartida. ARS Medica Rev Ciencias Médicas. 2017;42(2):4-6.

31. Omonaiye O, Kusljic S, Nicholson $\mathrm{P}$, Manias E. Medication adherence in pregnant women with human immunodeficiency virus receiving antiretroviral therapy in sub- Saharan Africa: a systematic review. BMC Public Health. 2018;18(805):1-20.

32. Gourlay A, Birdthistle I, Mburu G, Iorpenda K, Wringe A. Review article Barriers and facilitating factors to the uptake of antiretroviral drugs for prevention of mother-tochild transmission of HIV in subSaharan Africa: a systematic review. J Internactional AIDS Soc. 2013;16:18588.

33. Bozorgmehr K, Razum O. Effect of Restricting Access to Health Care on Health Expenditures among AsylumSeekers and Refugees: A QuasiExperimental Study in Germany, 19942013. PLoS One. 2015;10(7):e0131483.

34. Eziefula A, Sa M, Brown M. The health of recent migrants from resource- poor countries. Medicine (Baltimore). 2014;42(2):112-7.

35. Remme M, Siapka M, Vassall A, et al. The cost and cost-effectiveness of genderresponsive interventions for HIV: a systematic review. J Internactional AIDS Soc. 2014;17:19228.

36. Wu Hupat E. Human immunodeficiency virus infection in children and adolescents, 30 years in Chile. Vol. 89, Revista Chilena de Pediatria. Sociedad Chilena de Pediatria; 2018. p. 660-8.

37. MINSAL. Guía clínica AUGE: síndrome de la inmunodeficiencia adquirida VIH/ SIDA [Internet]. Ministerio de Salud; 2014 [cited 2020 Jun 12]. Available from: http://www.repositoriodigital.minsal.cl/ handle/2015/497.

38. Ministerio de Salud de Chile. Norma Conjunta de Prevención de la Transmisión Vertical del VIH y la Sífilis.
Programa Nacional de Prevención y Control del VIH/SIDA e ITS, editor. Santiago de Chile; 2012.

39. Ministerio de Salud. Programa Nacional de Prevención y Control del VIH/SIDA e ITS. [Internet]. Santiago; 2019. Available from: www.minsal.cl.

40. CONASIDA \& MINSAL. Boletín epidemiológico semestral VIH/SIDA. Junio del 2000. Serie documentos CONASIDA No. 12. ISSN 0717-411X. 2000.

41. Ministerio de Salud de Chile. Política de Salud de Migrantes Internacionales en Chile. Polit Salud Migrantes Int. 2017;

42. Cabieses B, Libuy M, Dabanch J, Santiago M. Hacia una comprensión integral de la relación entre migración internacional y enfermedades infecciosas: De la creencia a la evidencia para la acción sanitaria en Chile [Internet]. Santiago; 2019 [cited 2020 Jun 14]. Available from: https:// repositorio.udd.cl/handle/11447/2813.

43. Cabieses B. Health of migrants: simple questions can improve care. Lancet. 2019 Jun 8;393(10188):2297-8.

44. Sepúlveda C, Cabieses B. Rol del facilitador intercultural para migrantes internacionales en centros de salud chilenos: perspectivas de cuatro grupos de actores clave. Rev Peru Med Exp Salud Publica [Internet]. 2019 Dec 6 [cited 2020 Jun 14];36(4):592-600. Available from: https://rpmesp.ins.gob.pe/index.php/ rpmesp/article/view/4683.

45. Donati AP, Rubinstein MM, Lagos DV. Intervention with haitian mothers living with HIV/AIDS. Vol. 90, Revista Chilena de Pediatria. Sociedad Chilena de Pediatria; 2019. p. 356-7. 